\title{
Análisis de la velocidad adoptada en corredores de Maratón Analysis of the pacing strategies used by Marathon runners
}

\author{
*José Joaquín Díaz-Martín, **Eduardo J. Fernández-Ozcorta, ***Pablo Floría, *Jordan Santos-Concejero \\ * Universidad del País Vasco (España), **Universidad de Huelva (España), ***Universidad Pablo de Olavide (España)
}

Resumen: El propósito de este estudio fue analizar la influencia del nivel de rendimiento y sexo sobre los ritmos de carrera adoptados por atletas en tres maratones con perfiles muy parecidos en cuanto a recorrido y condiciones ambientales. Se analizaron 14420 atletas, 13387 hombres y 1034 mujeres. Los resultados indicaron que existían diferencias entre sexos en todos los tramos. Asimismo, hubo diferencias entre grupos con diferentes niveles de rendimiento, tanto en hombres como en mujeres. También se encontraron diferencias en las velocidades de cada tramo entre los diferentes ritmos empleadas en ambos sexos (54\% hombres y 50\% mujeres), siendo las mayores diferencias encontradas entre el primer y último tramo. Por lo general, el ritmo más utilizado durante la competición es aquel en que el atleta ralentiza su velocidad en el transcurso de los $42 \mathrm{~km}$. Este estudio ha demostrado que independientemente del sexo y el rendimiento, los atletas adoptan en su gran mayoría un ritmo positivo para completar un maratón. Palabras clave: ritmo, rendimiento físico, carrera, sexo.

Abstract: The purpose of this study was to analyze the influence of gender and athletic status on race pacing strategies used by runners in three marathons with similar profiles in terms of itinerary and environmental conditions. A total of 14420 athletes were analyzed, 13387 men and 1034 women. The results indicated that there were pacing differences between men and women in all sections. Furthermore, differences between groups of different athletic status in both men and women were observed. The biggest differences were found in the first and the final section. In general, the pacing strategy that athletes used the most (54\% in men and 50\% in women) was positive. This study shows that regardless of gender and performance level, the majority of athletes adopt a positive pacing strategy to complete a marathon.

Keywords: pacing, physical endurance, running, gender.

\section{Introducción}

Uno de los aspectos más importante que influye en el rendimiento del maratón es la estrategia adoptada durante la competición (Renfree \& Gibson, 2013). El éxito de las carreras, no depende sólo de haber entrenado y preparado adecuadamente con antelación, sino también de la estrategia que elige el atleta durante la competición, independientemente de su capacidad (Hanley, 2014). Aunque puede existir poca diferencia fisiológica entre competidores, los atletas pueden ganar o perder dependiendo de la capacidad de gastar adecuadamente la energía para prevenir la fatiga prematura antes de la finalización del evento (Garland, 2005; Hanley, 2014; Skorski \& Abbis, 2017). En este sentido, la estrategia de distribución del ritmo mucho más eficiente desde un punto de vista energético en los últimos records mundiales conseguidos (Díaz, Fernández-Ozcorta \& Santos-Concejero, 2018).

La influencia de la estrategia a lo largo de la prueba del maratón ha tenido gran interés en la literatura científica. En concreto, entender cómo distribuir el ritmo puede llevar a una mejor comprensión de los factores que influyen en el rendimiento y ayudar a los corredores a completar un maratón con éxito o a mejorar su rendimiento en carrera (Abbiss \& Laursen, 2008; Díaz, et al., 2018).

Diferentes estrategias se han identificado previamente en varios eventos de resistencia: ritmo positivo (donde el atleta ralentiza su velocidad en el transcurso de la carrera), ritmo negativo (el atleta acelera) y ritmo constante (se mantiene una velocidad relativamente constante) (Hanley, 2013). La adopción de un ritmo u otro va a depender de la capacidad del atleta en mantener la homeostasis (Abbiss \& Laursen, 2008). En el caso de seleccionar el ritmo equivocado podrían aparecer síntomas como la fatiga que ralentizaría la velocidad del corredor, llegando incluso a provocar el abandono de la carrera. Desde la literatura se sugiere que el ritmo negativo es el más adecuado para un ejercicio prolongado como el maratón. Este ritmo negativo lleva a una menor reducción del agotamiento de los hidratos de carbono, menor consumo excesivo de oxígeno y a la concentración de lactato en sangre, pero este perfil de ritmo no siempre es adoptado en la práctica (Hanley, 2013). En cuanto a las concentraciones de lactato, existen trabajos que predicen, a través de la cinética del lactado, el rendimiento atlético en la prueba estudiada (Muñoz Pérez, Moreno Pérez, Cardona González, \& EsteveLanao, 2012).

Fecha recepción: 21-02-18. Fecha de aceptación: 07-07-18 Eduardo J. Fernández-Ozcorta eduardo.fernandez@dempc.uhu.es
Pese a que el ritmo negativo puede ser el más recomendable, el ritmo más utilizado por la gran mayoría de los corredores de maratón es el positivo, ralentizando la velocidad conforme avanza la carrera, sobre todo después de 20-25 km de carrera(Ely, Martin, Cheuvront \& Montain, 2008; Santos-Lozano, et al., 2014). Aunque existen diferencias si se comparan las estrategias utilizadas entre corredores de diferente nivel de rendimiento y sexo (Deaner, Carter, Joyner \& Hunter, 2015; March, Vanderburgh, Titlebaum, \& Hoops, 2011; Santos-Lozano, et al., 2014). Estudios recientes han observado que los mejores corredores poseen una menor variabilidad en su velocidad de carrera en comparación con los atletas menos exitosos (Lamber, et al., 2004; Ely, et al., 2008; Renfree \& Gibson, 2013; Santos-Lozano, et al., 2014). Esta diferencia de utilización de ritmo no es solo observada entre grupos corredores de diferente de rendimiento, sino también entre sexos. Las mujeres, con respecto a los hombres, suelen utilizar velocidades que pueden mantener durante toda la carrera, a diferencia de los hombres, quienes habitualmente realizan una última parte de la carrera a menor velocidad que la primera (Deaner, et al., 2014; Trubee, Vanderburgh, Diestelkamp \& Jackson, 2014).

Aunque es conocido que el sexo y el nivel de rendimiento influyen en el tipo de estrategia usada durante el maratón (Deaner, et al. 2014; March, et al., 2011), poco se sabe sobre cómo se distribuyen cada tipo de estrategia entre los corredores de maratón en función de su sexo y nivel de rendimiento. Un análisis más profundo de los tipos de estrategias utilizadas por los corredores de maratón podría ayudar a aumentar el conocimiento sobre esta prueba, con el fin de que entrenadores y atletas puedan elegir estrategias enfocadas a mejorar el rendimiento.

Por lo expuesto ahora, a pesar de que el maratón es una carrera popular que convoca a miles de corredores, existen pocos estudios que analicen las estrategias adoptadas en función del nivel de rendimiento de los corredores. Además, las diferencias observadas entre sexos, insta a explorar dichas diferencias en diferentes grupos de rendimiento. Por lo tanto, el objetivo del presente estudio fue analizar la distribución de las estrategias utilizadas durante el maratón en hombres y mujeres en diferentes niveles de rendimiento. Según los estudios precedentes, se partió de la hipótesis de que las diferencias entre hombres y mujeres fueron más acusadas en los niveles de rendimiento más altos.

\section{Método}

\section{Participantes}

La muestra estuvo compuesta por 14420 atletas, donde el $92.8 \%$ ( $\mathrm{n}=13386)$ eran hombres, mientras que el 7.8\% $(\mathrm{n}=1034)$ fueron mujeres. Se analizaron los resultados de tres maratones disputados en Sevilla, en los años 2013 y 2014, y en Castellón en el año 2013. Aunque se 
analizaron dos maratones con recorridos diferentes, ambos tuvieron perfiles muy parecidos y condiciones ambientales (temperatura $4.0 \pm$ $1.7^{\circ} \mathrm{C}$, humedad $86 \pm 5 \%$, desnivel acumulado $242 \pm 8 \mathrm{~m}$ ).

\section{Procedimiento}

El estudio incluyó el análisis de los datos disponibles para el público, por lo que el consentimiento informado individual no fue necesario. Los resultados se obtuvieron mediante la descarga de la «clasificación general absoluta» a través de la empresa Cronochip.com. Cada conjunto de datos incluye al menos: los datos de posición, categoría, tiempo oficial y pasos (10 km, $21.1 \mathrm{~km}$ y $30 \mathrm{~km}$ ).

La distancia total se dividió en cuatro tramos de $10 \mathrm{~km}, 21.1 \mathrm{~km}, 30$ km y $42.195 \mathrm{~km}$. Se calculó individualmente la velocidad media total y la velocidad media de cada tramo. Por otra parte, el ritmo se definió como el porcentaje de la variación en la velocidad durante cada tramo en relación con la velocidad media total de cada corredor. Los participantes fueron divididos en función del ritmo de carrera en tres grupos: «ritmo constante» (mantiene la misma velocidad durante toda la carrera), «ritmo positivo» (el atleta ralentiza la velocidad en el transcurso del evento) y «ritmo negativo» (el atleta aumenta progresivamente la velocidad durante toda la carrera). Para diferenciar a cada grupo, se calculó la ratio entre la velocidad del primer tramo y del cuarto tramo. Cuando los datos se encontrasen entre 1.05 y .95 se consideró un ritmo constante. Asimismo, si la ratio fue mayor a 1.05 se consideró como ritmo negativo, mientras que si fue menor a .95 se consideró como ritmo positivo.

Los participantes fueron divididos en cuatro grupos de rendimiento dependiendo del tiempo final de la prueba: grupo 1 [corredores muy rápidos, el tiempo en finalizar la prueba d» 202 min. (hombres) y d» 226 min. (mujeres)], grupo 2 [corredores rápidos, el tiempo en finalizar la prueba 203 a $222 \mathrm{~min}$. (hombres) y 227 a $247 \mathrm{~min}$. (mujeres)], grupo 3 [corredores medianos, el tiempo en finalizar la prueba 223 a $243 \mathrm{~min}$ (hombres) y 248 a 271 min. (mujeres)], y el grupo 4 [corredores lentos, tiempo en finalizar la prueba $>244 \mathrm{~min}$. (hombres) y > $272 \mathrm{~min}$. (mujeres)]. Para obtener grupos homogéneos, el criterio utilizado fue dividir la muestra en cuartiles (primer, segundo, tercero y cuarto cuartil) un 25\% del tiempo neto final de todos los finalistas de cada maratón.

\section{Análisis estadístico}

El análisis estadístico realizado presento diferentes etapas. La primera etapa fue el cálculo descriptivo, la homocedasticidad y la normalidad en la distribución de la muestra. Para este ello se las variables fueron testadas mediante las pruebas de Levene y Kolmogorov-Smirnov, respectivamente. En un segundo paso, con el objeto de evaluar y comparar tanto las diferencias entre grupos de rendimiento como las diferencias entre grupos en función del ritmo adoptado durante la competición, se utilizó el análisis de la varianza (ANOVA). Además, se calculó el porcentaje de varianza explicada por medio del indicador omega cuadrado (ù $\left.{ }^{2}\right)$. Este indica la proporción de varianza entre grupos que es explicada por las diferencias en las velocidades empleadas. Finalmente, se calculó el tamaño del efecto (TE) grupo a grupo para determinar la magnitud de las diferencias (Cohen, 1977). Asimismo, para evaluar la influencia del nivel de rendimiento sobre los ritmos empleados se utilizaron tablas de contingencia con el estadístico Chi cuadrado.

El estudio estadístico se realizó con software estadístico empleado fue el SPSS (vers. 20), empleando un nivel de significación estadística fijado en $p<.05$.

\section{Resultados}

Para evaluar y estudiar las estrategias empleadas por los participantes del maratón se analizaron los tiempos de pasos en horas, minutos y segundos (hh:mm:ss), de las tres pruebas estudiadas (Tabla 1).

La velocidad media normalizada en cada tramo, analizando las diferencias entre hombres y mujeres se muestra en la Figura 1 . Se observaron diferencias entre sexos en todos los tramos, aunque estás fueron pequeñas o triviales $(\mathrm{t}>3.78, p<.05$, $\mathrm{TE}<0.31$ ). Los hombres lograron velocidades superiores en todos los tramos exceptuando en el último donde las mujeres alcanzaron una mayor velocidad.
Por lo general, la estrategia más utilizada durante la competición por hombres y mujeres fue la positiva, una disminución progresiva de la velocidad durante el transcurso de la prueba (Tabla 2). Aunque en las mujeres se observó una mayor utilización de las estrategias constante y negativa en comparación con los hombres $\left(X^{2}=11.19\right.$, df $\left.=2, p<.01\right)$.

En ambos sexos (Tabla 3), se encontraron diferencias en la distribución de las estrategias empleadas entre los grupos de rendimiento analizados $\left(X^{2}>171\right.$, df $\left.=6, p<.01\right)$. En concreto, la utilización de la estrategia constante más usada, tanto en hombres como en mujeres, conforme el rendimiento aumentaba, observándose una mayor utilización en atletas muy rápidos en comparación con los corredores más lentos en ambos sexos. La estrategia de ir incrementando la velocidad a lo largo de la prueba, «la negativa» fue la menos habitual de las analizadas. Se observó una mayor frecuencia de uso en los grupos de rendimiento de atletas rápidos (grupo 2) y medios (grupo 3). En los dos sexos, el grupo de rendimiento que con mayor frecuencia adoptó una estrategia positiva fue el lento, mientras que en los grupos de atletas rápidos y muy rápidos se observó un menor porcentaje de utilización con respecto a los grupos de menor rendimiento.

Tabla 1

Análisis descriptivo de los tiempos de paso (hh:mm:ss)

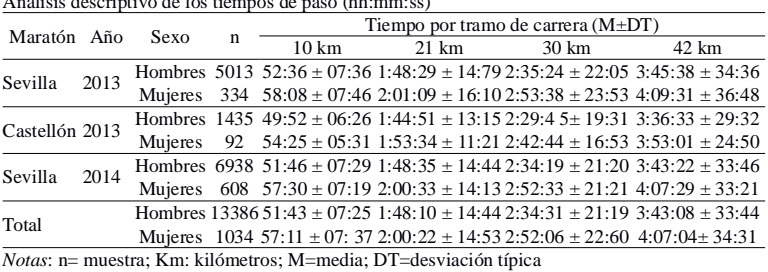

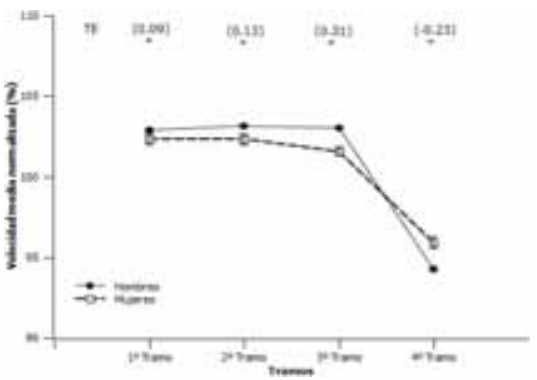

Felocidad media normalizada ( $\$ intervalo de confianza al 95\%) en cada tramo .

Tabla 2

Distribución de las estrategias empleadas por sexos

\begin{tabular}{ccccccc}
\multicolumn{5}{c}{ Hombres } & \multicolumn{5}{c}{ Mujeres } & \multirow{2}{*}{ Diferencias $X^{2}$} \\
\hline Positivo & Constante & Negativo & Positivo & Constante & Negativo & $11.19 \dagger$ \\
54 & 36 & 10 & 50 & 38 & 12 & \\
\hline
\end{tabular}
\begin{tabular}{cccc}
54 & 36 & 10 & 50 \\
\hline Denota diferencias de distribución entre sexos.
\end{tabular}

Tabla 3.

Distribución de las estrategias empleadas por grupos de rendimiento y sexos

Grupos de $\quad$ Hombres $\quad$ Mujeres $\quad$ Diferencias entre

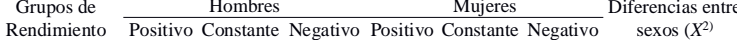

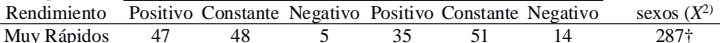

$\begin{array}{llllllll}\text { Muy Rápidos } & 47 & 48 & 5 & 35 & 51 & 14 & 287 \dagger \\ \text { Rápidos } & 40 & 44 & 16 & 32 & 49 & 19 & 354 \dagger \\ \text { Medi } & 52 & 34 & 14 & 50 & 36 & 14 & 57 \dagger\end{array}$

$\begin{array}{cccccccc}\text { Rápidos } & 40 & 44 & 16 & 32 & 49 & 19 & 354 \dagger \\ \text { Medio } & 52 & 34 & 14 & 50 & 36 & 14 & 57 \dagger\end{array}$

\begin{tabular}{llllllll} 
Medio & 52 & 34 & 14 & 50 & 36 & 14 & $57 \dagger$ \\
Lentos & 77 & 18 & 5 & 82 & 15 & 3 & $791 \dagger$ \\
\hline
\end{tabular}

Diferencias entre 1289* $171^{*}$

gotas: * Denota diferencias de distribución entre grupos de rendimiento; †Denota diferencias de distribución entre sexos.

\section{Discusión}

El objetivo del presente trabajo fue analizar la distribución de las estrategias utilizadas durante el maratón en hombres y mujeres en diferentes niveles de rendimiento. Según los datos encontrados, se partió de la hipótesis teorizó que las diferencias entre hombres y mujeres fueron más acusadas en los niveles de rendimiento más altos.

Entre los hallazgos principales encontrados, se observó que tanto los hombres como las mujeres adoptaron velocidades iniciales altas que no lograron mantener durante la competición. Estos datos indicaron que, por lo general, se empleaban como estrategia de carrera un ritmo positivo, siendo la segunda parte de la carrera más lenta que la primera. Está información coincide con Santos-Lozano et al. (2014), que observaron cómo los corredores maratonianos adoptaron un ritmo positivo, 
disminuyendo la velocidad de carrera a medida que transcurría la competición, independientemente del sexo y del nivel de rendimiento. Según Santos-Lozano et al. (2014), está disminución en los corredores más rápidos competitivos puede ser explicada por diversas razones. Primero porque los corredores no reservan energía para los últimos kilómetros de la prueba. En segundo lugar, por el resultado final del maratón, ya que por lo general no se define en los últimos kilómetros. Y tercero, porque el grupo de cabeza se reduce gradualmente en número, y sólo los mejores posicionados son capaces de mantener el ritmo.

Además, el análisis mostró cómo los hombres lograron velocidades superiores en todos los tramos excepto en el último, donde las mujeres alcanzaron una mayor velocidad, aunque la magnitud de las diferencias fue pequeña. En relación a estos resultados, Trubee et al. (2014) observaron que las mujeres tienden a exhibir una disminución menos brusca de la velocidad de carrera que los hombres durante las últimas etapas del maratón. March et al. (2011) entienden que las mujeres no élite son consistentemente mejores marcando el ritmo en relación a los hombres no élite, incluso después de controlar la edad, sexo y tiempo de finalización. Diversos mecanismos explican porque las mujeres podrían ser más rápidas que los hombres en el último tramo de carrera. Según Speechly et al. (1996), las mujeres son capaces de rendir a un mayor porcentaje de su consumo máximo de oxígeno ( $\mathrm{VO}_{2}$ máx.) que los hombres en los últimos tramos de una prueba de gran fondo. Otros estudios (e.g., Carter, et al., 2001; Ruby \& Roberts, 1994; Tarnopolsky, 2000) observan que las mujeres tienen un índice de intercambio respiratorio inferior quelos hombres duranteel ejercicioderesistencia submáxima, lo que sugiere una preferencia por la oxidación de las grasas para obtener energía mientras ahorran glucógeno. Esto a su vez, puede retrasar el «muro» del corredor, un fenómeno en el que las reservas de glucógeno en el cuerpo se agotan en el último cuarto de carrera (Coyle, 2007; Ruby \& Roberts, 1994; Tarnopolsky, 2000), contribuyendo así a la disminución precipitada de la velocidad de carrera.

Por otra parte, los ritmos adoptados por los atletas participantes en este estudio revelaron que el más utilizado por todos los grupos de rendimiento fue el positivo, seguido del «constante» y el «negativo». Estos resultados coinciden con los reportados por Santos-Lozano et al. (2014), quienes observaron en diferentes ediciones del maratón de Nueva York (2006-2011), que todos los grupos de rendimiento seleccionaron un perfil de ritmo positivo. En esta misma línea fueron los resultados de Ely et al. (2008). Por los datos obtenidos en la presente investigación, se observó que, con independencia del sexo y el nivel de rendimiento, los atletas por lo general disminuyeron sus velocidades progresivamente durante la segunda mitad del maratón adoptando un ritmo positivo.

A pesar de que los datos indican que el ritmo positivo fue el más frecuente en todos los grupos de rendimiento, la distribución de los porcentajes en cada ritmo fue diferente en cada grupo de rendimiento. En el grupo de atletas más rápidos se observó un porcentaje más elevado de atletas que utilizaban un ritmo «constante» en comparación con el resto de grupos de rendimiento. Santos-Lozano et al. (2014) indicaron que los mejores atletas poseen una menor variabilidad en la velocidad de carrera en comparación con los atletas menos exitosos. Del mismo modo, Nikolaidis y Knetchtle(2017a, 2017b) y Nikolaidis, Onywera y Knetchtle (2018) descubrieron cómo los corredores más rápidos del Maratón de New York adoptan un ritmo más uniforme que el resto. Por otro lado, Renfree et al. (2012) observaron que las primeras clasificadas en el maratón del Campeonato del Mundo del 2009 adoptaron un patrón de velocidad más constante durante toda la competición, a diferencias de los corredores menos exitosos, que seleccionaron velocidades insostenibles con el resultado de una pérdida significativa de velocidad.

Según los resultados encontrados, en el presente estudio, en el grupo de atletas rápidos (grupo 2) y medios (grupo 3), se observó un porcentaje más elevado de atletas que utilizaba un ritmo negativo en comparación con el resto de grupos. El uso de este tipo de ritmo se señala en la literatura como el más adecuada para un ejercicio prolongado como es el maratón, debido a la menor reducción del agotamiento de los hidratos de carbono, menor consumo excesivo de oxígeno y menor concentración de lactato en sangre (Hanley, 2013). Por último, en el grupo de los atletas más lentos, el porcentaje de atletas que utilizaron un ritmo positivo fue mayor en comparación con el resto de grupos. La disminución de la velocidad en deportes de larga resistencia se debe a diversos factores como la regulación del ritmo, que es dictado en gran medida por la capacidad de resistir la fatiga (Abbiss \& Laursen, 2008; Haney \& Mercer, 2011), por un proceso de aprendizaje (Lambert et al., 2004) y por la temperatura, además de otras variables como la experiencia o el nivel de entrenamiento (Knechtle, et al., 2015; Trubee, et al., 2014).

De los datos obtenidos en la presente investigación se observa que, independientemente del sexo, los corredores más lentos mostraron mayores diferencias respecto al resto de grupos de rendimiento. Los atletas más lentos adoptaron un ritmo más rápido que su velocidad media durante los primeros $21 \mathrm{~km}$, para después reducir drásticamente su velocidad de carrera, por debajo de la velocidad media total. Las mayores diferencias se observaron en el último tramo. Está información coincide con otros estudios como el de Renfree y Gibson (2013) y Santos-Lozano et al. (2014) quienes encontraron resultados similares, mostrando que los corredores más lentos exhibieron una mayor disminución en la velocidad de carrera en comparación con los mejores corredores. Por otro lado, Lambert et al. (2004) observaron que los atletas ultra maratonianos (corredores participantes en carreras de $100 \mathrm{~km}$ ) más rápidos regulan su velocidad de manera más eficaz en comparación con los atletas más lentos, que disminuyeron su velocidad significativamente a partir de la segunda mitad de la competición. Además, los corredores más rápidos (grupo 1) tuvieron menos cambios de velocidad y fueron capaces de mantener la velocidad inicial durante una mayor distancia que los corredores más lentos.

Según Hanley (2013), una de las posibles razones por la que la mayoría de los atletas adoptan ritmos insostenibles es porque tratan de seguir el ritmo marcado por los líderes, con el objetivo de mantener ese ritmo durante el mayor tiempo posible para colocarse en una buena posición y mejorar su rendimiento anterior. Otra posible explicación a la reducción drástica de la velocidad de carrera, y que puede ser el resultado de un patrón o un ritmo inadecuado, subyace en el agotamiento del glucógeno, hipoglucemia o hipertermia (Coley, 2007). Aunque la desaceleración en el maratón podría estar relacionada con haber entrenado y preparado con antelación la carrera (formación previa), desde el punto de vista fisiológico Joyner et al. (2011) indicaron que una relación entre el $\mathrm{VO}_{2}$ máx y la economía de carrera podría retrasar el agotamiento de glucógeno y reducir la producción de calor metabólico, reduciendo así el riesgo de hipertermia. Asimismo, en la segunda parte de la carrera, dos factores importantes entran en juego y son determinantes para explicar la disminución de la velocidad de carrera y la adaptación de un ritmo u otro. Por un lado, la homeostasis, donde el cerebro podría coordinar subconscientemente una respuesta de regulación para asegurar que la intensidad del ejercicio no supere los límites fisiológicos del cuerpo (Abbis \& Laursen, 2008; Edwards, et al., 2011; Faulkner, et al., 2008). Por otro lado, una percepción menor de esfuerzo en los corredores más rápidos gracias a una mayor experiencia a sufrir síntomas asociados al ejercicio (Faulkner, et al., 2008).

En general, la presente investigación genera aspectos importantes para atletas de maratón élites y no élites, y para otros deportistas que participan en competiciones similares, ya que el ritmo adoptado tiene una gran influencia en el resultado final. Si los atletas, independientemente del nivel del rendimiento, son capaces de adoptar el ritmo correcto durante la competición y, además, comprender los factores que influyen en el rendimiento, serán capaces de registrar tiempos más rápidos e incluso terminar por delante de otros corredores con capacidades fisiológicas superiores que utilizaron ritmos menos eficaces, completando con éxito y mejorando su rendimiento en el maratón.

A pesar de ello, la presente investigación presenta limitaciones que han de ser consideradas. El número de tramos analizados fueron únicamente cuatro, siendo estos de una longitud de alrededor de $10 \mathrm{~km}$. Analizar tramos más cortos podría permitir observar cambios de velocidad más frecuentes, así como una definición de los ritmos empleados por los corredores más precisa.

Por los datos obtenidos en la presente investigación, se ha demos- 
trado que, con independencia del sexo y el nivel de rendimiento, los atletas adoptan en su gran mayoría un ritmo positivo para completar un maratón. Este ritmo positivo está caracterizado por correr el último tramo más lento que los precedentes. La velocidad empleada en el último tramo fue la que determinó en mayor medida las diferencias entre grupos de rendimiento. Todos los grupos mostraron una caída de la velocidad en el último tramo, pero ésta fue más acusada en los corredores lentos en comparación con el resto de grupos.

Se concluye que el presente trabajo puede aportar información importante tanto para atletas de maratón de cualquier nivel como para otros deportistas que participen en competiciones de características similares, ya que el ritmo adoptado tiene una gran influencia en el resultado final. Aquellos atletas, independientemente del nivel del rendimiento, que sean capaces de adoptar el ritmo correcto durante la competición y además comprender los factores que influyen en el rendimiento, serán capaces de registrar tiempos más rápidos, e incluso terminar por delante de otros corredores con capacidades fisiológicas superiores que utilizaron ritmos menos eficaces, completando con éxito y mejorando su rendimiento en el maratón.

\section{Referencias}

Abbiss, C. R., \& Laursen, P. B. (2008). Describing and understanding pacing strategies during athletic competition. Sports Medicine, 38(3), $239-52$.

Baden, D., Warwick-Evans, L., \& Lakomy, J. (2004). Am I nearly there? The effect of anticipated running distance on perceived exertion and attentional focus. Journal of Sport and Exercise Psychology, 26(2), 215-231.

Carter, S. L., Rennie, C., \& Tarnopolsky, M. A. (2001). Substrate utilization during endurance exercise in men and women after endurance training. American Journal of Physiology Endocrinology and Metabolism, 280(6), E898-E907.

Cohen, J. (1977). Statistical Power Analysis for the Behavioral Sciences. New York, NY: Academic Press.

Coyle, E. F. (2007). Physiological regulation of marathon performance. Sports Medicine, 37(4-5), 306-311.

DeKoning, J. J., Foster, C., Bakkum, A., Kloppenburg, S., Thiel, C., Joseph, T., Cohen, J., \& Porcari, J. P. (2011). Regulation of pacing strategy during athletic competition. Plos ONE, 6(1), e15863. doi: 10.1371/journal.pone.0015863.

Deaner R. O., Carter R. E, Joyner M. J, \& Hunter S. K. (2015). Men are more likely than women to slow in the marathon. Medicine and Science in Sports and Exercise, 47(3), 607-616. doi: 10.1249/ MSS.0000000000000432.

Díaz, J. J., Fernández-Ozcorta, E. J., \& Santos-Concejero, J. (2018). The influence of pacing strategy on marathon world records. European journal of sport science, 1-6. doi: 10.1080/17461391.2018.1450899

Edwards, A. M., Bentley, M. B., Mann, M. E., \& Seaholme, T. S. (2011) Self-pacing in interval training: a teleoanticipatory approach. Psychophysiology, 48(1), 136-141. doi: 10.1111/j.14698986.2010.01034.x.

Ely, M. R., Martin, D. E., Cheuvront, S. N., \& Montain, S. J. (2008). Effect of ambient temperature on marathon pacing is dependent on runner ability. Medicine and Science in Sports and Exercise, 40(9), 1675-1680. doi: 10.1249/MSS.0b013e3181788da9.

Faulkner, J., Parfitt, G., \& Eston, R. (2008). The rating of perceived exertion during competitive running scales with time. Psychophysiology, 45(6), 977-985. doi:10.1111/j.14698986.2008.00712.x.

Foster, C., Schrager, M., Snyder, A. C. \& Thompson, N. N. (1994) Pacing strategy and athletic performance. Sports Medicine, 17(2), 77-85.

Foster, C., deKoning, J. J., Hettinga, F., Lampen, J. Dodge, C., Bobbert, M., \& Porcari, J. P. (2004). Effect of competitive distance on energy expenditure during simulated competition. International Journal of Sports Medicine, 25(3), 198-204.

Garland, S. (2005). An analysis of the pacing strategy adopted by elite competitors in $2000 \mathrm{~m}$ rowing. British Journal of Sports Medicine, 39(1), 39-42. doi: 10.1136/bjsm.2003.010801.

Hanley, B. (2013). An analysis of pacing profiles of world-class racewalkers. International Journal of Sports Physiology and Performance, 8(4), 435-441.

Hanley, B. (2014). Senior men's pacing profiles at the IAAF World Cross Country Championships. Journal of Sports Sciences, 32(11), 1060-
1065. doi: 10.1080/02640414.2013.878807.

Haney, T., \& Mercer, J. (2011). A description of variability of pacing in marathon distance running. International Journal of Exercise Science, 4(1), 133-140

Joyner, M. J., Ruiz, J. R., \& Lucia, A. (2011). The two-hour marathon: who and when? Journal of Applied Physiology, 110(1), 275-277. doi: 10.1152/japplphysiol.00563.2010.

Knechtle, B., Stiefel, M., Rosemann T., Rüst C., \& Zingg, M. (2015). Running and the association with anthropometric and training characteristics. Therapeutische Umschau, 72(5), 343-355. doi:10.1024/0040-5930/a000685.

Lambert, M., Dugas, J., Kirkman, M., Mokone, G., \& Waldeck, M. (2004). Changes in running speeds in a $100 \mathrm{~km}$ ultra-marathon race. Journal of Sports Science \& Medicine, 3(3), 167-173.

March, D. S., Vanderburgh, P. M., Titlebaum, P. J., \& Hoops, M. L. (2011). Age, sex, and finish time as determinants of pacing in the marathon. Journal of strength and conditioning research. 25(2), 386-391. doi: 10.1519/JSC.0b013e3181bffd0f.

Muñoz Pérez, I., Moreno Pérez, D., Cardona González, C., \& EsteveLanao, J. (2012). Prediction of race pace in long distance running from blood lactate concentration around race pace. Journal of Human Sport and Exercise, 7(4), 763-769. doi: 10.4100/jhse.2012.74.04

Nikolaidis, P. T., \& Knechtle, B. (2017a). Do fast older runners pace differently from fast younger runners in the «new york city marathon»? Journal of Strength and Conditioning Research. [Epub ahead of print]. doi: 10.1519/JSC.0000000000002159

Nikolaidis, P. T., \& Knechtle, B. (2017b). Effect of age and performance on pacing of marathon runners. Open Access Journal of Sports medicine, 21(8), 171-180. doi: 10.2147/OAJSM.S141649

Nikolaidis, P. T., Onywera, V. O., \& Knechtle, B. (2017). Running Performance, Nationality, Sex, and Age in the 10-km, Half-Marathon, Marathon, and the 100-km Ultramarathon IAAF 1999-2015. Journal of Strength and Conditioning Research, 31(8), 2189-2207. 10.1519/JSC.0000000000001687

Nummela, A., Keränen, T., \& Mikkelsson, L. O. (2007). Factors related to top running speed and economy. International Journal of Sports Medicine, 28(8), 655-661.

Renfree, A., \& Gibson, A. (2013). Influence of different performance levels on pacing strategy during the women's World Championship marathon race. International Journal of Sports Physiology and Performance, 8(3), 279-285.

Ruby, B. C., \& Robergs, R. A. (1994). Gender differences in substrate utilization during exercise. Sports Medicine, 17(6), 393-410.

Santos-Lozano, A., Collado, P. S., Foster, C., Lucia, A., \& Garatachea, N. (2014). Influence of Sex and Level on Marathon Pacing Strategy. Insights from the New York City Race. International Journal of Sports Medicine, 35(11), 933-938. doi: 10.1055/s-0034-1367048.

Skorski, S., \& Abbiss, C.R. (2017). The Manipulation of Pace within Endurance Sport. Frontiers in Physiology, 27(8), 102. doi: 10.3389/ fphys.2017.00102

Speechly, D. P., Taylor, S. R., \& Rogers, G. G (1996). Differences in ultraendurance exercise in performance-matched male and female runners. Medicine and Science in Sports and Exercise, 28(3), 359-365.

St. Clair Gibson, A., Lambert, E. V., Rauch, L. H., Tucker, R., Baden, D. A., Foster, C., \& Noakes, T. D. (2006). The role of information processing between the brain and peripheral physiological systems in pacing and perception of effort. Sports Medicine, 36(8), 705-722.

Tarnopolsky, M. A. (2000). Gender differences in substrate metabolism during endurance exercise. Canadian Journal of Applied Physiology, 25(4), 312-327.

Thiel, C., Foster, C., Banzer, W., \& de Koning J. (2012). Pacing in Olympic track races: competitive tactics versus best performance strategy. Journal of Sports Sciences, 30(11), 1107-1115. doi: 10.1080/ 02640414.2012.701759.

Thompson, P. (2007). Perspectives on coaching pace skill in distance running: a commentary. International Journal of Sports Science \& Coaching, 2(3), 219-221. doi: 10.1260/174795407782233128.

Townsend, M. (1982). Road-racing strategies. Medicine and Science in Sports and Exercise, 14(3), 235-243.

Trubee, N. W., Vanderburgh, P. M., Diestelkamp, W. S., \& Jackson, K. J. (2014). Effects of Heat Stress and Sex on Pacing in Marathon Runners. Journal of Strength and Conditioning Research, 28(6), 1673-1678. doi: 10.1519/JSC.0000000000000295.

Tucker, R. (2009). The anticipatory regulation of performance: the physiological basis for pacing strategies and the development of a perception-based model for exercise performance. British Journal of Sports Medicine, 43(6), 392-400. doi: 10.1136/bjsm.2008.050799. 